use of modelling is particularly effective, and gets around the practical difficulties of directly observing mechanochemical reactions at atomic resolution using microscopy.

Finally, the work points to a future in which an informed understanding of mechanochemistry will improve the prediction and design of reactions. This would provide a more rational basis for the field and facilitate the application of mechanochemical reactions as a mainstream synthetic method. .

Stuart L. James is in the School of Chemistry and Chemical Engineering, Queen's University Belfast, Belfast BT9 5GE, UK.

e-mail:s.james@qub.ac.uk
1. Yan, H. et al. Nature 554, 505-510 (2018).

2. Takacs, L. Chem. Soc. Rev. 42, 7649-7659 (2013).

3. Faraday, M. Q. J. Sci. Lit. Arts 8, 374-376 (1820).

4. Lea, M. C. Am. J. Sci. 43, 527-531 (1892).

5. May, P. A. \& Moore, J. S. Chem. Soc. Rev. 42, 7497-7506 (2013).

6. James, S. L. et al. Chem. Soc. Rev. 41, 413-447 (2012).

\section{Force-activated ion channels in close-up}

\section{Piezo proteins allow cells to sense forces by letting ions pass through the cell membrane in response to mechanical stimuli. Three structures of a Piezo protein shed light on how this crucial process works. SEE ARTICLES P.481 \& P.487}

$\mathrm{A}$ 11 cells can sense mechanical force, but it is not clear exactly how. It is known, however, that two closely related members of the mammalian Piezo family of membrane proteins, Piezo1 and Piezo2, have key roles in many physiological and developmental processes that involve mechanosensitivity, including touch, breathing and vascular development ${ }^{1}$. Two papers in Nature, by Saotome et al. ${ }^{2}$ (page 481) and Zhao et al. ${ }^{3}$ (page 487), and a third published in eLife by Guo and MacKinnon ${ }^{4}$, now report structures of Piezol from the mouse (mPiezo1), obtained using a technique known as cryogenic electron microscopy (cryo-EM). The structures provide insight into how Piezo proteins might sense and respond to mechanical force.

It has been difficult to identify mechanically activated channels - proteins that enable a cell to sense force by allowing ions to pass through the cell membrane in response to mechanical stimuli. A major advance in this field was the discovery of the mechanosensitive Piezo channels ${ }^{5}$, which are found in many invertebrates, vertebrates and plants. The mPiezo1 protein is unusually large compared with most proteins, consisting of 2,547 amino-acid residues, and is structurally unrelated to most other proteins.

A previously reported cryo-EM study ${ }^{6}$ provided the first insight into the architecture of mPiezo1. It revealed that the channel is a homotrimer (constructed from three identical subunits) consisting of three 'propeller blades' around a central pore. However, the relatively low resolution of the structure allowed only 14 out of the 38 transmembrane helices in each subunit to be resolved, and none of the amino-acid side chains.

The latest cryo-EM structures ${ }^{2-4}$ have significantly higher resolution (about 4 ångströms) and reveal many crucial structural features of mPiezo1. The channel is a triskelion, with each arm (or propeller blade) consisting of nine repeated structural units, each containing four transmembrane $a$-helices. These arms surround a central pore formed by the two transmembrane $\alpha$-helices (known as TM37 and TM38) nearest to the protein's carboxyl terminus (Fig. 1).

For each arm, the structures of the three repeating units (which consist of TM25 to
TM36) nearest the pore were obtained at higher resolution than the next three units (which consist of TM13 to TM24). Each arm bends at the junction between these two trios of repeating units. Viewed from the extracellular side of the channel, the bend angle is about $100^{\circ}$, giving the channel the appearance of a clockwise spiral. When the trimer is viewed from the side, the bend angle is about $140^{\circ}$, so that the channel looks like an inverted dome. Analyses of the amino-acid sequence of mPiezol reported in the three new studies suggest that three additional repeat units (which consist of TM1 to TM12) form from the amino-terminal sequences of the protein and comprise the tip of each arm. However, these distal repeat units could not be resolved in the cryo-EM structures, perhaps because they are too flexible.

There are several notable structural features near the centre of the channel. On the extracellular side, there is a large cap on top of the central pore. On the cytoplasmic side, an $\alpha$-helix acts as a long 'beam' that connects TM28 to the central pore region. This beam might be involved in the mechanism by which force opens and closes the pore (the

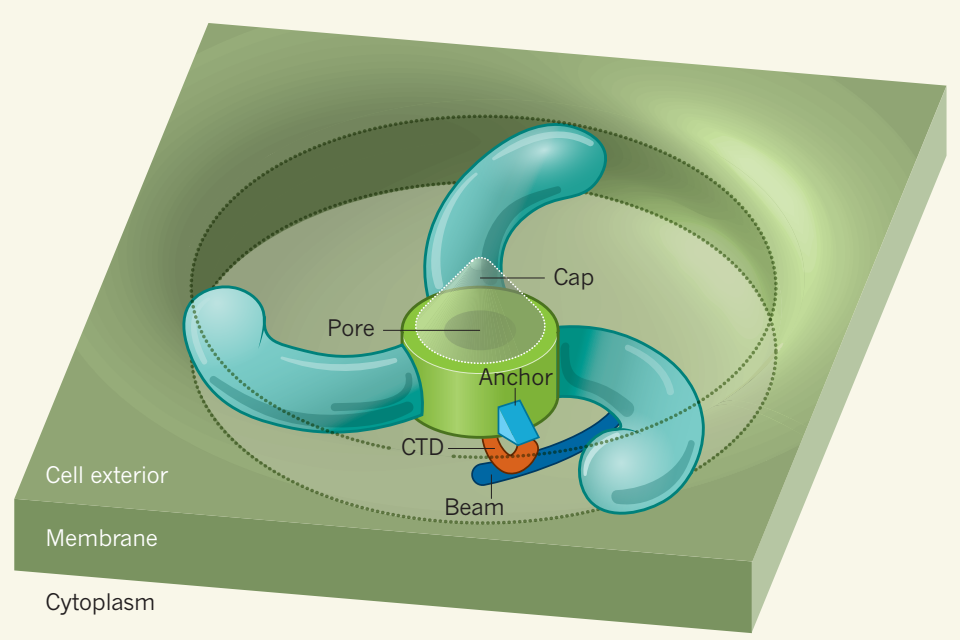

Figure 1 | Cartoon of the mechanosensitive mPiezol channel. Three papers ${ }^{2-4}$ report the structure of the mouse Piezo1 channel (mPiezo1), a membrane protein that responds to mechanical stimuli by opening its channel, thus allowing external ions to pass through the cell membrane into the cytoplasm. The channel forms from three mPiezol proteins, and consists of three bent arms surrounding a central pore, which has an extracellular cap. The cytoplasmic carboxy-terminal domain (CTD) of each mPiezol molecule extends from the pore to contact an anchor domain and a 'beam' that connects the arm to the pore. For simplicity, only one CTD, anchor and beam are shown. Guo and MacKinnon ${ }^{4}$ propose that the curvature of the arms induces deformation of the cell membrane; the membrane would fill the regions between the arms, but is not shown here for simplicity. 
force-gating mechanism), along with several other domains (dubbed the latch, clasp and anchor) and the carboxy-terminal domain (CTD), which extends from the 'inner helix' formed by the pore-lining TM38. Saotome et al. and Zhao et al. performed mutagenesis experiments in which they replaced amino acids in these key regions with other amino acids to observe the effects on channel behaviour. These structure-based mutagenesis studies provide insight to the machineries involved in ion permeation through the channel and force gating.

The long central pore of mPiezol seems to connect to the aqueous surroundings of the channel through lateral portals that serve as ion-access pathways; additional windows possibly open to the membrane interior. These features are reminiscent of the structures of trimeric acid-sensing ion channels and P2X receptors ${ }^{7}$. Zhao et al. report that negatively charged amino-acid residues in the lateral portals influence the ion selectivity and other properties of the pore, supporting the notion that the portals form part of the ionpermeation pathway.

Also notable is Saotome and colleagues' finding that a phenylalanine residue at a pore constriction affects both permeation and the rate at which the channel is inactivated when subjected to a prolonged stimulus. As pointed out by Saotome et al. and Zhao et al., inactivation is also modified by disease-causing mutations of multiple charged residues that are involved in the interactions of the anchor with the pore-lining inner helix and the CTD. Taken together with a recent biophysical study ${ }^{8}$ that suggests the existence of intricate connections between the ion-permeation pathway and channel inactivation, the structures of mPiezol provide hints about the coupling between the pore and the machinery that opens the channel in response to force.

Additional structure-guided studies using mutagenesis and electrophysiology are now needed to further investigate the function of mPiezo1. Other approaches, such as moleculardynamics simulations, should help to reveal the force-gating mechanisms. Zhao and colleagues' findings, along with those of others ${ }^{8,9}$, suggest that multiple parts of mPiezol are involved in force gating, including its $\mathrm{N}$-terminal region. A more complete structure that includes the currently unresolved 1,100 amino-acid residues at the N-terminal end of the protein is therefore needed. Moreover, the reported structures seem to show a closed channel. The structure of an open channel could provide valuable clues to the permeation and gating mechanisms.

Guo and MacKinnon observed that the unusual dome shape of the mPiezol trimer causes deformation of synthetic membrane vesicles. They suggest that the curvature of the arms induces deformation of the cell membrane that changes with membrane tension and on channel opening. It will be interesting to see whether mPiezol-induced membrane deformation is detectable in vivo, and how it responds to mechanical stimuli.

Apart from mPiezo1, structural information is available for three other families of bona fide mechanically activated channels. The molecules and gating mechanisms all seem to be distinct. The bacterial MscL protein is a homopentamer, and responds to membrane tension by opening a wide pore ${ }^{10}$. The dimeric TRAAK and TREK channels have windows that might allow lipid fatty-acid chains to extend into the pore, and they respond to membrane tension by simultaneously opening the channel and expanding the area of the membrane that is occupied by the channel, although the tension range needed for channel activation differs from that of $\mathrm{MscL}^{11}$. The homotetrameric NompC channel found in the fruit fly Drosophila contains a bundle of four helices that looks like a coiled spring; this is thought to tether the channel to microtubule structures in the cytoskeleton for force gating $^{12}$. It is to be hoped that some general principles that unite these seemingly disparate structures will emerge as we learn more about each system.

Yuh Nung Jan and Lily Yeh Jan are at the Howard Hughes Medical Institute, Departments of Physiology and Biochemistry, University of California, San Francisco, San Francisco, California 94158, USA. e-mails:yuhnung.jan@ucsf.edu; lily.jan@ucsf.edu

1. Murthy, S. E., Dubin, A. E. \& Patapoutian, A. Nature Rev. Mol. Cell. Biol. 18, 771-783 (2017).

2. Saotome, K. et al. Nature 554, 481-486 (2018)

3. Zhao, Q. et al. Nature 554, 487-492 (2018).

4. Guo, Y. R. \& MacKinnon, R. elife 6, e33660 (2017).

5. Coste, B. et al. Science 330, 55-60 (2010).

6. Ge, J. et al. Nature 527, 64-69 (2015).

7. Kellenberger, S. \& Grutter, T. Mol. Biol. 427, 54-66 (2015).

8. Moroni, M., Servin-Vences, M. R., Fleischer, R. \& Lewin, G. R. Preprint at bioRxiv http://dx.doi. org/10.1101/156489 (2017).

9. Wu, J., Goyal, R. \& Grandl, J. Nature Commun. 7, 12939 (2016)

10.Kung, C. Nature 436, 647-654 (2005)

11.Brohawn, S. G. Ann. N. Y. Acad. Sci. 1352, 20-32 (2015).

12.Jin, P. et al. Nature 547, 118-122 (2017)

This article was published online on 12 February 2018. injury or the loss of one copy of the gene Nr5a2. This state might predispose mice, and possibly humans, to pancreatitis and pancreatic cancer. SEE LETTER P.533

\section{CHARLES MURTAUGH \\ \& RAYMOND J. MACDONALD}

I nflammation can initiate the repair of injured tissue, but, if it persists, can also be a fertile ground for disease and cancer ${ }^{1}$. For instance, chronic inflammation is central to pancreatitis, in which digestive enzymes produced by the pancreas's acinar cells are inappropriately activated, causing tissue digestion and cell death ${ }^{2}$. In mice, transient downregulation of acinar-cell-specific transcription factors and their targets aids recovery from pancreatitis, perhaps by reducing further enzyme production ${ }^{3}$. But irreversible downregulation of these factors is associated with tumours in mice $e^{4-6}$, and people with pancreatitis have an increased risk of pancreatic cancer ${ }^{2}$. Until now, the causal link between pancreatic inflammation, gene-expression changes and cancer has not been understood. On page 533, Cobo et al. ${ }^{7}$ describe how repurposing of the pancreatic transcription factor $\mathrm{Nr} 5 \mathrm{a} 2$ controls the acinar-cell inflammatory response.

The same research group had previously discovered $^{8}$ that loss of one of the two copies (alleles) of the Nr5a2 gene in mice impairs pancreatic regeneration after mild inflammation, and sensitizes acinar cells to a cancercausing protein. It seemed likely, therefore, that decreased levels of $\mathrm{Nr} 5 \mathrm{a} 2$ diminish the resilience of pancreatic cells.

In the current study, Cobo et al. explored this theory by analysing NR5A2-protein levels in samples from humans with a type of cancer called pancreatic ductal adenocarcinoma (PDAC). Consistent with their theory, the researchers found that NR5A2 levels in tumour cells tended to be low in people who had a history of pancreatitis. These patients were also more likely to carry a common single-nucleotide mutation linked to the NR5A2 gene, associated with increased risk of PDAC, than were people whose tumour cells had high levels of NR5A2. These observations support the idea that low NR5A2 levels increase PDAC risk by raising the 